\title{
A Simple Method for Removal of the Distal Segment of a Broken Femoral Intramedullary Nail: A Case Report
}

\author{
KL Pan, FRCS(Glasg), Reuben PR*, MD \\ Department of Orthopaedics, Universiti Malaysia Sarawak, Kuching, Malaysia \\ *Department of Orthopaedics, Sarawak General Hospital, Kuching, Malaysia
}

\begin{abstract}
We report a method for removing the distal segment of a broken locked intramedullary nail secondary to nonunion of the femur following radiotherapy for fibromatosis. A Kirschner wire with one tip fashioned into a hook was used to remove the distal segment without opening with the nonunion site. Details of the procedure are described.
\end{abstract}

Key Words:

Broken intramedullary nail, removal of distal segment

\section{INTRODUCTION}

Intramedullary nails used for femur fixation will eventually break if the fracture does not unite. When increasing callus formation is seen on radiographs, indicating the healing process is ongoing, an exchange nailing can be performed as a defnitive treatment. This is best conducted without interfering with the callus and overlying soft tissues. We report a patient where removal of the distal segment of the nail was done without opening up the fracture site, using a long Kirschner wire.

\section{CASE REPORT}

A 34-year old woman was admitted with a broken locked femoral intramedullary nail. She had a history of right thigh fibromatosis with multiple excisions (four times) and recurrences over a period of five years. After the second and fourth excision, she received 25 fractions of radiotherapy, adding up to a total of 50 fractions. One year later, she sustained a pathological fracture at the isthmus of the femur for which an interlocking Targon nail was inserted. The bone did not unite despite bone grafting. This nail subsequently broke, as did a second nail which was inserted followng the first refracture. The current admission was for the third broken nail.

Plain radiographs showed a broken nail at the non-union site with exuberant callus around it. We considered this a hypertrophic nonunion and planned for an exchange nailing without intefering with the callus that had already formed.

For surgery, the patient was placed in the standard supine position using the interlocking nail traction table with imageintensifier access. An incision was made through the old proximal scar over the greater trochanter. The locking screw was removed followed by the proximal broken nail segment and the distal locking screws. Next, a small acute-angled "hook" was fashioned at the end of a long $2.0 \mathrm{~mm}$ Kirschner wire. This wire was $30 \mathrm{~cm}$ long and was the longest available in our operating theatre. It would not have reached the distal end of the broken nail but was sufficient to reach the distal screw holes. This was more easily accomplished by bending a longer segment at the end and then cutting off the tip to create a small hook. (Figures 1a, 1b) The other end of the Kwire was held by a T-handle and inserted in an antegrade fashion into the distal segment via the trochanteric opening. At the screw- hole site, the hook was oriented to catch onto the hole. This required a few attempts while rotating the wire using the T-handle. When the hook "caught" the hole, a mallet was used to knock on the T-handle to retrogradely remove the distal segment through the trochanteric opening. We did not push through the distal end of the nail because the available K-wire was too short and also the tapered end of the nail would not have allowed the hooked end to go through. (Figures 1c, 1d, 1e, 1f) Another similar nail was reinserted without difficulty and the patient's recovery was uneventful.

\section{DISCUSSION}

Broken intramedullary nails in the presence of non-union are often removed via the non-union site. This necessitates a new skin opening, excision of callus, stripping of periosteum and angulation of the non-union site. All these interfere with the blood supply to a region where the circulation is already tenuous, a major factor in non-union in the first place. This patient had undergone multiple surgeries and received a maximal dose of radiotherapy, thereby resulting in creation of a fibrotic region with sparse blood supply and subsequent 


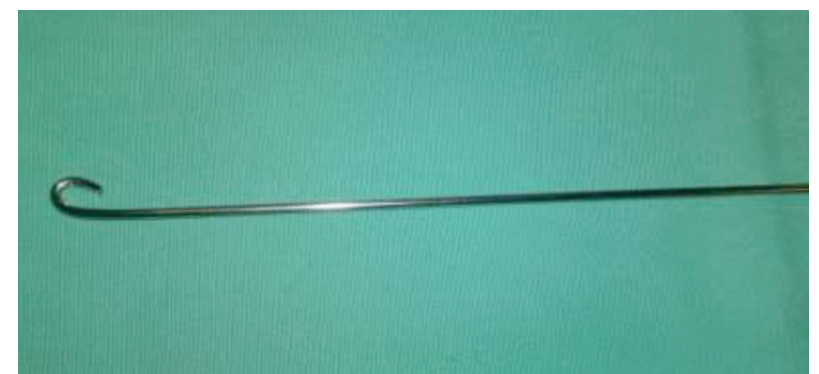

Fig. 1a: K-wire with the tip bent.

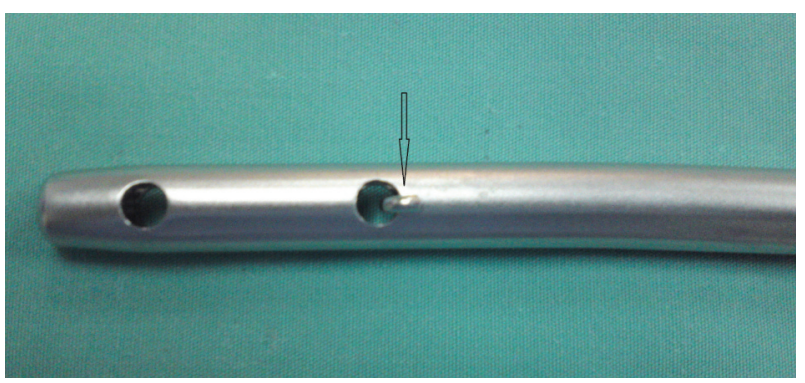

Fig. 1c: Tip of wire engaging screw hole with arrow indicating the curved tip.

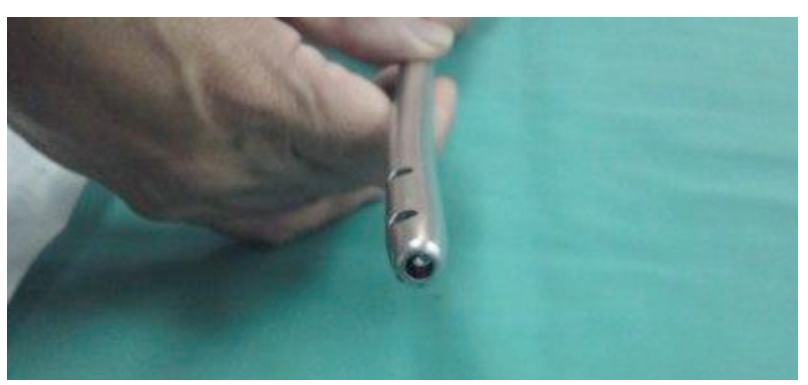

Fig. 1e: Tapered end of nail would not have allowed a curved Kwire to go through.

non-union. Without union, the stress on the nail ultimately resulted in a fatigue fracture and this was now the third time that the nail had broken. However, the latest x-ray showed good callus formation. Our aim was to provide stability with an exchange nailing without interfering with the union process and avoiding surgery at the non-union site.

Several methods have been described for the removal of broken intramedullary nails. Franklin et $\mathrm{al}^{1}$ used a custom made hook to extract the distal fragment in 56 patients. Different sizes of the hook were available for the different sized nails. Metikala ${ }^{2}$ described eight patients who underwent closed retrograde retrieval of the distal broken segment using a ball-tipped guide wire. The technique involved using a standard plain guide wire passed through the cannulated distal broken nail segment after extraction of the proximal nail fragment. The plain guide wire was advanced distally into the knee joint under fluoroscopy imaging. Over this wire, a 5-millimeter $(\mathrm{mm})$ cannulated

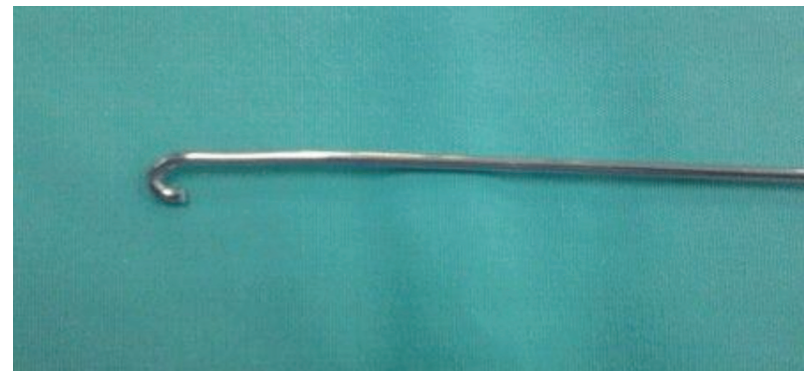

Fig. 1b: Same wire with distal 2-3mm cut off to make it easier to engage the screw holes.

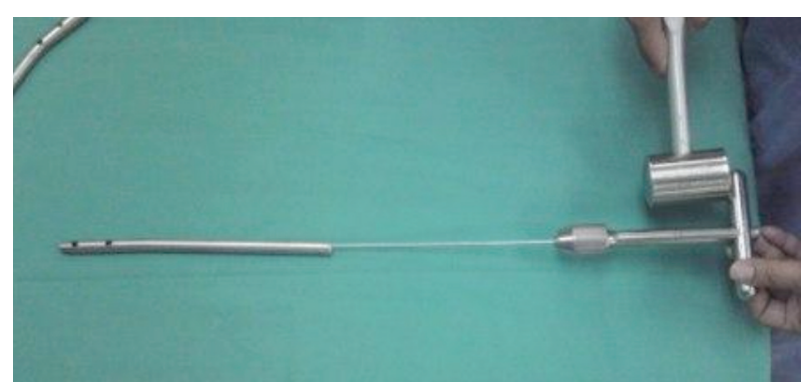

Fig. 1d: T-handle holding the K-wire and a mallet to help deliver the broken nail proximally.

large drill bit was used to create a track up to the distal broken nail segment. Through the small knee wound, a balltipped guide wire was passed, smooth end first, till the ball engaged the end of the nail. The guide wire was then extracted along with the broken nail through the proximal wound. Cabrita ${ }^{3}$ reported five patients and used a technique involving the application of an $\mathrm{AO}$ distractor prior to removal of the nail fragments, with subsequent removal of the proximal nail fragment in an anterograde fashion and removal of the distal fragment through a medial parapatellar approach. Impaction of the fracture site was then accomplished with a nail that was broader than the remaining fragmented material. The disadvantage of the above methods is the necessity for opening the knee joint.

Georgilas ${ }^{4}$ used a $10.5 \mathrm{~mm}$ reamer, which was passed over a guide wire into the intramedullary canal and wedged into the distal broken fragment. The nail was removed gently using rotatory movements under fluoroscopic guidance. Blake ${ }^{5}$ used an olive tipped guide wire which was passed completely through the nail. The proximal part was then removed using a standard extraction equipment. Next, a second, non-olive tipped, wire was passed through the distal fragment adjacent to the previously placed wire. The olive tipped wire was then withdrawn, causing it to impinge against the non-tipped wire and the outer tip of the nail. A Thandled chuck was applied to the proximal end of the olive tipped wire and this was gently tapped back bringing with it, the distal fragment. However, a small tapered nail may not allow two guide wires to protrude through the distal end simultaneously. 
In the present case, we made use of tools readily available in an orthopaedic operating room. We had no difficulty in removing the distal fragment as it was loose and the break was at the isthmus of the femur. If it was distal to the isthmus, the proximal part of the femur would possibly had to be reamed to a larger diameter after removing the proximal nail segment. It is possible to insert two or more hooked K-wires to latch onto the available screw holes, making the extraction process more likely to succeed. These wires can be held tightly together by a single T-handle chuck before using a mallet to tap the T-bar to remove the nail. Possible limitations are the lack of availability of a long Kwire and if the distal fragment has a tight fit on the surrounding cortical bone. This method adds to the existing methods that a surgeon can use to remove the distal end of a broken nail without interfering with the non-union site.

\section{REFERENCES}

1. Franklin JL, Winquist RA, Benirschke SK, Hansen ST. Jr Broken intramedullary nails. J Bone Joint Surg Am. 1988; 70: 146371.

2. Metikala S, Mohammed R. Closed retrograde retrieval of the distal broken segment of femoral cannulated intramedullary nail using a ball-tipped guide wire. Indian J Orthop. 2011; 45(4): 347-50.

3. de Amorim Cabrita HA, Malavolta EA, Teixeira OV, Montenegro NB, Duarte FA, Mattar R Jr. Anterograde removal of broken femoral nails without opening the nonunion site: a new technique. Clinics (Sao Paulo). 2010; 65(3): 279-83.

4. Georgilas I, Mouzopoulos G, Neila C, Morakis E, Tzurbakis M. Removal of broken distal intramedullary nail with a simple method: a ccase report. Arch Orthop Trauma Surg. 2009; 129(2): 203-5.

5. Blake SM. A technique for the removal of the distal part of a broken intramedullary nail. Ann R Coll Surg Engl. 2009; 91(2): 169-70. 